\title{
A role for mitogen-activated protein kinase activation by integrins in the pathogenesis of psoriasis
}

\author{
Ingo Haase, Robin M. Hobbs, M. Rosario Romero, Simon Broad, and Fiona M. Watt \\ Keratinocyte Laboratory, Imperial Cancer Research Fund, London, United Kingdom \\ Address correspondence to: Fiona M. Watt, Keratinocyte Laboratory, Imperial Cancer Research Fund, \\ 44 Lincoln's Inn Fields, London WC2A 3PX, United Kingdom. \\ Phone: 44-20-7269-3528; Fax: 44-20-7269-3078; E-mail: watt@icrf.icnet.uk.
}

Ingo Haase's present address is: Department of Dermatology, University of Cologne, Cologne, Germany. M. Rosario Romero's present address is: Prolifix Ltd., Abingdon, United Kingdom.

Ingo Haase and Robin M. Hobbs contributed equally to this work.

Received for publication January 5, 2001, and accepted in revised form May 29, 2001.

\begin{abstract}
In normal epidermis, $\beta 1$ integrin expression is confined to the basal layer, whereas in hyperproliferative epidermis, integrins are also expressed in the suprabasal layers. Transgenic mice in which integrins are expressed suprabasally via the involucrin promoter have a sporadic psoriatic phenotype; however, the mechanism by which integrins contribute to the pathogenesis of psoriasis is unknown. We observed activation of mitogen-activated protein kinase (MAPK) in basal and suprabasal keratinocytes of human and transgenic mouse psoriatic lesions and healing mouse skin wounds, correlating in each case with suprabasal integrin expression. Phenotypically normal human and transgenic mouse epidermis did not contain activated MAPK. Transgene-positive keratinocytes produced more IL- $1 \alpha$ than controls did, and keratinocyte MAPK could be activated by ligation of suprabasal integrins or treatment with IL-1 $\alpha$. Constitutive activation of MAPK increased the growth rate of human keratinocytes and delayed the onset of terminal differentiation, recreating many of the histological features of psoriatic epidermis. We propose that activation of MAPK by integrins, either directly or through increased IL-1 $\alpha$ production, is responsible for epidermal hyperproliferation in psoriasis and wound healing, and that the sporadic phenotype of the transgenic mice may reflect the complex mechanisms by which IL-1 release and responsiveness are controlled in skin.
\end{abstract}

J. Clin. Invest. 108:527-536 (2001). DOI:10.1172/JCI200112153.

\section{Introduction}

The unique structure of the epidermis is maintained by a tightly regulated balance between keratinocyte proliferation and terminal differentiation. Stem cells within the epidermal basal layer self-renew and also produce nonstem daughters, known as transit-amplifying cells (1). Transit-amplifying cells divide a small number of times before commitment to terminal differentiation. Committed cells withdraw from the cell cycle, detach from the epidermal basement membrane, and move into the suprabasal layers of the epidermis. As they migrate through the suprabasal layers toward the skin surface, keratinocytes undergo striking changes in morphology and gene expression (2). In the first suprabasal layers, they enlarge; they express novel keratins and several proteins, including involucrin and cornifin, that are precursors of a structure known as the cornified envelope. Once they have entered the outermost layers, the cells flatten and lose their nucleus, and the envelope precursors become enzymatically crosslinked by transglutaminase 1 , resulting in envelope assembly. The cornified cells are continually shed from the surface of the skin and are replenished through proliferation of stem cells in the basal layer.

Attachment of basal keratinocytes to the basement membrane is mediated by integrin receptors, including the $\alpha 6 \beta 4$ integrin, which is a receptor for laminin, and several $\beta 1$ integrins, the most abundant of which are $\alpha 2 \beta 1$ (collagen receptor) and $\alpha 3 \beta 1$ (laminin receptor). In addition to their adhesive role, $\beta 1$ integrins regulate keratinocyte differentiation. Human epidermal stem cells have higher levels of $\beta 1$ integrins and are more adhesive to ECM proteins than are transit-amplifying cells $(1,3)$, and when a dominant negative $\beta 1$ integrin mutant is introduced into human keratinocytes in culture the proportion of transit-amplifying cells is markedly increased (4). When keratinocytes are deprived of contact with the ECM, both stem and transit-amplifying cells differentiate without further rounds of division (3); suspension-induced terminal differentiation can be inhibited with ECM proteins or antibodies to $\beta 1$ integrins, suggesting that integrin ligation is a negative regulator of terminal differentiation (5).

Given the role of integrins in regulating keratinocyte adhesion and differentiation, it is not surprising that integrin expression is altered in both benign and neoplastic keratinocyte disorders (6). In particular, although integrin expression is normally confined to the basal layer of the epidermis, suprabasal integrin expression is a feature of hyperproliferative epidermis, as found, for example, after wound closure or in lesions of the benign 
human skin disorder psoriasis (6). That suprabasal integrin expression can play a causal role in the onset of psoriasis has been demonstrated by creating transgenic mice in which various integrin subunits are expressed under the control of the involucrin promoter $(7,8)$. In these mice, sporadic epidermal hyperproliferation with accompanying histological features of psoriasis, including a lymphocytic infiltrate, is observed.

There are two alternative hypotheses for how suprabasal integrin expression could trigger epidermal hyperproliferation: either the presence of integrins on the surface of suprabasal cells provides a signal that the underlying basal cells respond to, or the integrins signal to the suprabasal cells themselves, possibly leading to unscheduled synthesis of growth factors or cytokines (7, 8). Basal keratinocytes are not stimulated to proliferate when exposed to integrin-positive suprabasal keratinocytes in vitro (8); therefore, there is currently no evidence in favor of the first hypothesis. This has led us to consider the alternative hypothesis and to examine signal transduction by suprabasal integrins.

The classic mitogen-activated protein kinase (MAPK) cascade has previously been implicated in integrin regulation of keratinocyte terminal differentiation (4). The cascade consists of a module of three protein kinases acting in a hierarchical order: the MAP kinases ERK (extracellularly regulated kinase) 1 and 2 , which are activated by different isoforms of MAP kinase kinases (MEK) through phosphorylation of conserved threonines and tyrosines within a TXY motif (9), and the MAP kinase kinase kinases, including the Raf family, which regulate MAP kinase kinase (MAPKK) activity by phosphorylation of serine residues, such as serines 217 and 221 in MAPK kinase 1 (10). Expression of constitutively activated MAPKK (with glutamic acid substitutions at serines 217 and 221; known as MAPKK1; ref. 11) can overcome the effect of a dominant negative $\beta 1$ integrin mutant on human keratinocytes, thereby restoring the size of the stem cell compartment in vitro (4). In addition, a dominant negative MAPKK mutant (with an alanine substitution at serine 221 ; known as MANA; ref. 11) has the same effect as the dominant negative integrin, reducing keratinocyte adhesiveness and $\beta 1$ integrin levels and causing cells to leave the stem cell compartment (4). Given the role of $\beta 1$ integrin signaling via MAPK in the stem to transit-amplifying cell transition, we decided to investigate whether suprabasal integrins contribute to epidermal hyperproliferation by activating MAPK.

\section{Methods}

Source of tissue. Psoriatic lesions from the upper leg of nine untreated patients, and samples of normal human skin from the upper leg, back, and cheek of four patients who underwent tumor excisions, were kindly provided by K. Hartmann and M. Haitas-Haase (University of Cologne, Germany). Normal scalp skin and neonatal foreskin (one sample of each) were also examined. Skin samples were taken from the backs of transgenic mice expressing the human $\beta 1$ integrin subunit or human $\alpha 6 \beta 1$ under the control of the involucrin promoter (founder lines 0840 and 1137B; CBA X C57BL/10F1; refs. 7,8 ) and from littermate controls. In some experiments, 3-mm-diameter full thickness wounds were made in the backs of mice expressing the $\beta 1$ transgene or wildtype controls; $6-7$ days later, the animals were sacrificed and the wound sites collected for histological analysis.

Keratinocyte culture and retroviral infection. Normal human epidermal keratinocytes from neonatal foreskins (strains kq, km, and kn; passages 2-8) were cultured in FAD medium (one part Ham's F12 medium plus three parts DMEM and $0.18 \mathrm{mM}$ adenine) supplemented with $10 \%$ FCS and a cocktail of $0.5 \mu \mathrm{g} / \mathrm{ml}$ hydrocortisone, $5 \mu \mathrm{g} / \mathrm{ml}$ insulin, $10^{-10} \mathrm{M}$ cholera toxin, and $10 \mathrm{ng} / \mathrm{ml}$ epidermal growth factor (HICE) on a J23 T3 feeder layer as described previously (3). To determine the proportion of S-phase cells, keratinocytes were pulse-labeled with $10 \mu \mathrm{g} / \mathrm{ml} \mathrm{BrdU}$ for 1 hour and were harvested and immunolabeled as described previously (12). Organotypic cultures on de-epidermized dermis were carried out as described previously (13).

The constitutively active MAPK kinase 1 mutant, MAPKK1, with glutamic acid substitutions at serines 217 and 221, and the dominant negative mutant MANA, with an alanine substitution at serine 221 (11), cloned into pBabe puro, were generous gifts of C. Marshall (Institute of Cancer Research, London, United Kingdom). Keratinocytes were infected by coculture with retroviral producer cells as described previously (4).

Lines of murine keratinocytes derived from the skin of a transgenic mouse expressing human $\alpha 2 \beta 1$ or the human $\beta 1$ integrin subunit under the control of the involucrin promoter $(7,8)$ (passages 19-24) were cultured at $32^{\circ} \mathrm{C}$ on a J2-3T3 feeder layer in the same medium as used to culture human keratinocytes.

Immunostaining of cells and tissue sections. The following antibodies were used for immunohistochemistry: LH001 (monoclonal anti-human keratin 14; kindly provided by I. Leigh, London Hospital, London, United Kingdom), DH1 (rabbit anti-human involucrin), rabbit anti-Ki 67 (Novacastra, Newcastle, United Kingdom), rabbit antisera to doubly phosphorylated MAPK (NEB, Hitchin, United Kingdom, or MAPK-YT from Sigma Chemical Co., St. Louis, Missouri, USA), monoclonal anti-ERK2 (sc-1647 to stain human skin; Santa Cruz Biotechnology Inc., Santa Cruz, California, USA), rabbit anti-ERK2 (sc153 to stain mouse skin; Santa Cruz Biotechnology Inc.), mAb13 (monoclonal anti- $\beta 1$ integrin; Becton Dickinson, Lincoln Park, New Jersey, USA). Secondary antibodies labeled with Alexa 488 (green) and 594 (red) (Molecular Probes Inc., Eugene, Oregon, USA) were used. In some experiments, cell nuclei were stained using a DNA-specific dye, ToProIII (Molecular Probes Inc.) for 5-30 minutes at a concentration of $1 \mu \mathrm{M}$ (applied after incubation with secondary antibody). Controls were to stain with secondary antibody alone or to preincubate MAPK antibodies with blocking peptides.

Before staining, cultured cells and frozen sections of skin were fixed in $4 \%$ paraformaldehyde for 10 minutes 
at room temperature; cultured cells were then permeabilized with $0.4 \%$ Triton X 100 in PBS for 4 minutes at room temperature. Incubations with primary and secondary antibodies were for approximately 1 hour at room temperature with subsequent PBS washes, except in the case of anti-doubly phosphorylated MAPK antibodies, when the manufacturers' protocols were followed. Sections of paraformaldehyde-fixed, paraffinembedded day 11 organotypic cultures were stained with anti-Ki 67 , and the average number $( \pm$ SD) of positive cells in 12 high-power fields per section was determined. ELISAs of cytokine production. A line of murine keratinocytes expressing human $\alpha 2 \beta 1$ under the control of the involucrin promoter and a transgene negative control line (8) were grown to near confluence on a J2-3T3 feeder layer. Medium conditioned by the cultures overnight was collected, and then the feeder cells were removed and the keratinocytes harvested. Keratinocytes were plated for 8 or 24 hours onto agarose-coated dishes in FAD + fibronectin-free FCS + HICE to prevent cell attachment. After 8 hours in suspension, a fraction of the cells was collected and allowed to attach to dishes coated with 10 $\mu \mathrm{g} / \mathrm{ml}$ type IV collagen for a further 16 hours.

Both intracellular and secreted levels of cytokines were measured. To measure intracellular cytokines, cells were washed in PBS and lysed in 1\% NP40, $10 \mathrm{mM}$ iodoacetamide, $1 \mathrm{mM}$ EDTA, $1 \mu \mathrm{g} / \mathrm{ml}$ leupeptin, 1 $\mu \mathrm{g} / \mathrm{ml}$ pepstatin, and $0.4 \mathrm{mM}$ PMSF (14). IL- $1 \alpha, \mathrm{IL}-1 \beta$, TNF- $\alpha$, and IL- 6 levels in the collected samples were determined by use of ELISA kits (R\&D Systems Inc., Minneapolis, Minnesota, USA, and Amersham Pharmacia Biotech, Piscataway, New Jersey, USA).

Cell adhesion and MAPK phosphorylation. To analyze MAPK phosphorylation in response to integrin ligation in primary human keratinocytes, the cells were harvested with trypsin/EDTA and either suspended in serumfree medium supplemented with $10 \mathrm{ng} / \mathrm{ml}$ EGF for 30 minutes or plated at $37^{\circ} \mathrm{C}$ for various times onto bacteriological dishes coated with different ECM proteins or antibodies $(25 \mu \mathrm{g} / \mathrm{ml}$ fibronectin from Becton Dickinson; $50 \mu \mathrm{g} / \mathrm{ml}$ collagens type I and type IV from Sigma Chemical Co.; or $100 \mu \mathrm{g} / \mathrm{ml}$ mAb13) and blocked with $1 \%$ heat denatured BSA in PBS for 1 hour at $37^{\circ} \mathrm{C}$. Control dishes were coated with $20 \mu \mathrm{g} / \mathrm{ml}$ poly-L-lysine for 2 hours at $37^{\circ} \mathrm{C}$ and not blocked. Coating with laminin 5 was performed as described previously (4).

To assess the ability of IL- $1 \alpha$, IL-1 $\beta$, IL- 6 , and EGF to activate ERK1/2 and p38 MAPK in adherent primary human keratinocytes, dishes of preconfluent keratinocytes were starved overnight in serum-free medium after removing the feeders. Cells were then stimulated with FAD supplemented with cytokine or FAD alone for 15 minutes before harvesting. Recombinant human IL- 1 and IL- 6 were purchased from R\&D Systems Inc. and EGF from Peprotech (Rocky Hill, New Jersey, USA). To analyze modulation of MAPK phosphorylation by the MAPKK1 and MANA mutants, preconfluent keratinocytes were starved overnight after removing the feeders and then treated with FAD \pm FCS/HICE for 10 min- utes. To analyze MAPK phosphorylation in suspension, cells were held in FAD + FCS + HICE supplemented with methylcellulose (3) for 24 hours and then harvested.

The ability of suprabasal integrins to activate MAPK was determined using mouse keratinocytes expressing the human $\beta 1$ integrin subunit under the control of the involucrin promoter (8). The cells were held in suspension in FAD + FCS + HICE with methylcellulose for 16 hours, harvested, resuspended in FAD supplemented with $10 \mathrm{ng} / \mathrm{ml} \mathrm{EGF}$, and either held in suspension for 30 minutes or plated onto dishes coated with the human $\beta 1$ integrin-specific antibodies mAb13 or P5D2 (4) or with $25 \mu \mathrm{g} / \mathrm{ml}$ fibronectin.

Lysates of cultured keratinocytes were prepared and immunoblotted as described previously (4). In addition, lysates were prepared from a shaved biopsy of a psoriatic lesion and a control biopsy of normal human skin. Before extraction, the skin samples were frozen in liquid nitrogen and ground with a mortar and pestle. Thereafter, skin samples were processed in the same way as cultured cells. Activated MAPK was detected with antibodies specific for phosphorylated ERK1/2 or p38MAPK (NEB). Blots were reprobed with antibodies to ERK2 or p38MAPK (Santa Cruz Biotechnology Inc.). Protein bands were visualized with horseradish peroxidase-coupled secondary antibodies using enhanced chemiluminescence (ECL; Amersham, Buckinghamshire, United Kingdom).

Analysis of suspension-induced terminal differentiation. Suspension culture of human keratinocytes was carried out as described previously (3), except that the FCS used was depleted of fibronectin using Gelatin Sepharose 4B (Amersham Pharmacia Biotech, St. Albans, United Kingdom). Cells harvested from suspension culture were stained for flow cytometry with antibodies to involucrin (12), transglutaminase 1 (B.C1; gift of R. Rice, University of California, Davis, California, USA; ref. 15) and $\alpha$ cornifin (SQ37C; gift of A. Jetten, National Institute of Environmental Health Sciences, Research Triangle Park, North Carolina, USA; ref. 16) using essentially the method described for involucrin (12).

\section{Results}

MAPK is activated in byperproliferative keratinocytes. Nuclear translocation of the MAP kinases ERK1 and ERK2 is strictly dependent on enzyme activation (17). To confirm that nuclear localization was a reporter of MAPK activation in keratinocytes, we starved normal human epidermal keratinocytes overnight in FAD medium without serum or growth factors (HICE cocktail) and then treated the cultures for 15 minutes with FAD alone or FAD supplemented with $10 \%$ FCS and HICE before fixation. In serum-starved keratinocytes, there was no staining above background with an antibody specific for phosphorylated MAPK (ERK1,2) (Figure 1a). However, in cells treated with serum and growth factors, there was strong nuclear staining, and cytoplasmic staining was also increased (Figure 1b). A similar, though slightly less pronounced, translocation 

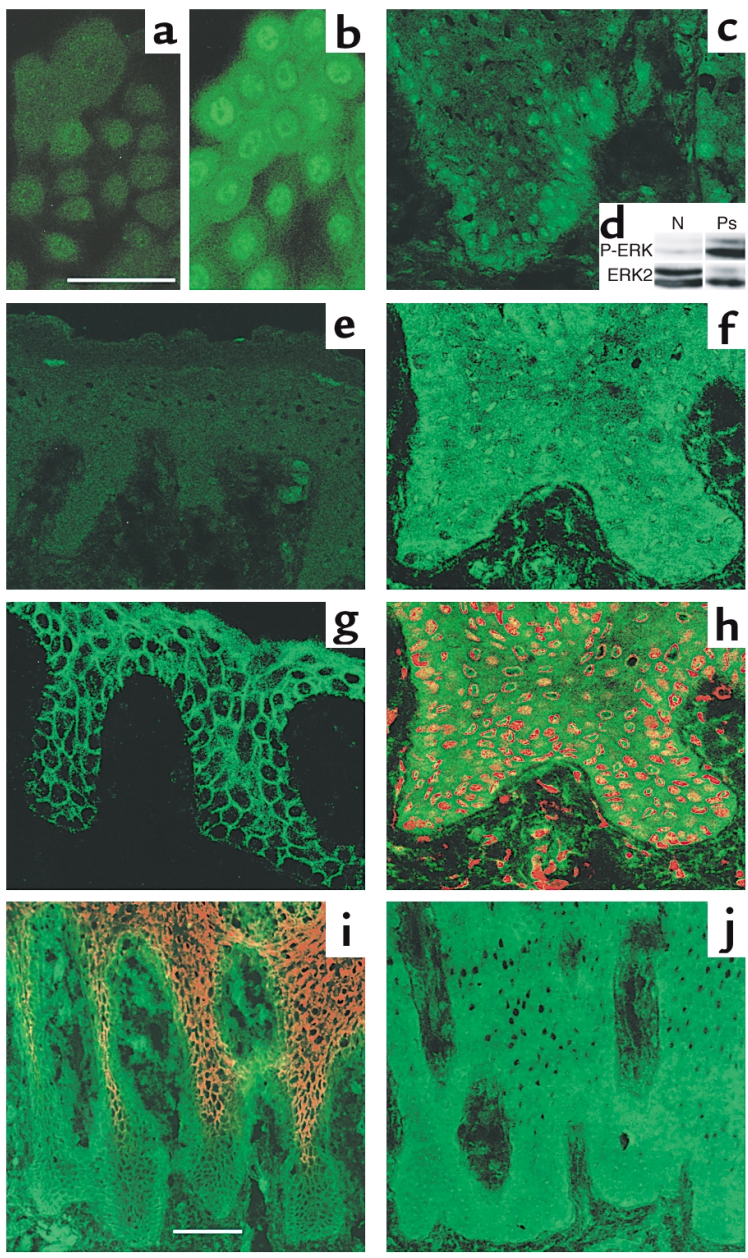

Figure 1

Distribution and activation of $\mathrm{p} 42^{\text {mapk }}$ in cultured keratinocytes and in normal and hyperproliferative human epidermis. Confocal immunofluorescence microscopy of growth factor-starved cultured keratinocytes (a) or keratinocytes treated with FCS + HICE for 15 minutes (b); psoriatic (c, $\mathbf{f}$, and $\mathbf{h}-\mathbf{j}$ ) or normal human epidermis (e and $\mathbf{g}$ ). Green staining in $\mathbf{a}-\mathbf{c}, \mathbf{e}-\mathbf{h}$, and $\mathbf{j}$ is for MAPK with antibody specific for activated ( $\mathbf{a}-\mathbf{c}$ and $\mathbf{e}$ ) or total MAPK ( $\mathbf{f}-\mathbf{h}$ and $\mathbf{j})$; red staining in $\mathbf{h}$ is nuclear dye, Topro III. The field shown in $\mathbf{h}$ is part of the field shown in f. (i) Staining for $\beta 1$ integrins (green) and involucrin (red). Scale bars: $50 \mu \mathrm{m}(\mathbf{a}, \mathbf{b}$, and $\mathbf{f}-\mathbf{h}), 100 \mu \mathrm{m}(\mathbf{c}, \mathbf{e}, \mathbf{i}$, and $\mathbf{j})$. (d) Western blot of shaved biopsies of normal human skin $(\mathrm{N})$ or psoriatic lesion $(\mathrm{Ps})$ probed with phosphorylation-specific antibody to ERK1/2 (P-ERK) or total ERK2.

of MAPK to the cell nucleus was observed with an antiERK2 antibody that detects both phosphorylated and unphosphorylated ERK2 (p42 $\left.{ }^{\text {mapk}}\right)$ (data not shown).

We next used immunofluorescence staining to examine MAPK activation in normal and hyperproliferative human epidermis. Frozen sections were stained with an $\mathrm{mAb}$ against total ERK2 or antibodies specific for phosphorylated MAPK. In healthy skin (from five individuals), the antibody to total MAPK predominantly stained the membrane region of epidermal keratinocytes with little cytoplasmic and no nuclear staining (Figure 1g), whereas anti-phospho-MAPK gave no staining above background (Figure 1e). In psoriatic epidermis (from eight individuals), there was both membranous and cytoplasmic staining with the antibody to total MAPK; in addition, the majority of cells in the basal and lower suprabasal layers showed a positive nuclear signal for MAPK (Figure 1, f, h, and j). Staining with the antibody to phosphorylated MAPK was predominantly nuclear in psoriatic lesions (Figure 1c). The suprabasal layers that had nuclear MAPK also showed strong expression of $\beta 1$ integrins (compare Figure 1i, green, with Figure $1 \mathrm{j}$, which is a serial section) and were further characterized by the absence of involucrin expression (Figure 1i, red). The enhanced activation of MAPK in psoriatic skin compared with normal skin was confirmed by Western blotting of shaved biopsies (Figure 1d).

Normal and hyperproliferative epidermis from transgenic mice that expressed $\beta 1$ integrins suprabasally under the control of the involucrin promoter (7) was stained with an antibody to ERK2 or anti-phosphoMAPK (Figure 2). In phenotypically normal epidermis from transgenic mice and transgene-negative littermate controls, there was weak cytoplasmic and plasma membrane staining and no nuclear staining with the antibody to total MAPK (Figure 2a and data not shown). The antibody to phospho-MAPK gave no staining above background (Figure 2c). In contrast, spontaneous hyperproliferative lesions from transgenic mice had pronounced nuclear phosphorylated MAPK (Figure 2d).

Epidermal hyperproliferation can be induced by wounding. We stained sections of hyperproliferative epidermis from $\beta 1$ transgenic mice and littermate controls 6 or 7 days after creating full thickness wounds. Immunostaining showed localization of ERK2 in the nuclei of many basal and suprabasal keratinocytes in the hyperproliferative area covering the wound (Figure $2 \mathrm{~b}$ ), but not in the adjacent nonaffected skin (data not shown). The nuclear localization of MAPK was confirmed by double staining with the DNA dye, Topro III (Figure 2b). The antibody to phosphorylated MAPK also gave strong nuclear staining of wounded, hyperproliferative epidermis (Figure 2e). Activation of MAPK was observed in wounds of wild-type and transgenic epidermis (Figure 2, b and e, and data not shown).

We conclude that MAPK was activated in hyperproliferative mouse and human epidermis, nuclear staining being observed in suprabasal integrin-positive cells. However, suprabasal integrin expression did not result in constitutive activation of MAPK, as no nuclear staining was observed in phenotypically normal epidermis from the transgenic mice.

Suprabasal integrins can activate MAPK. The MAPK cascade is a primary target of signaling pathways originating both from growth factor receptors and integrins, with growth factor-stimulated MAPK activity being dependent on integrin engagement $(18,19)$. In suspended primary human keratinocytes or keratinocytes plated onto the nonspecific adhesion substrate, poly-Llysine MAPK activity was low or undetectable, even in the presence of $10 \mathrm{ng} / \mathrm{ml} \mathrm{EGF}$ (Figure 3a). MAPK activation was observed upon engagement of $\beta 1$ integrins by collagen, fibronectin, and the $\beta 1$ integrin-specific antibody 

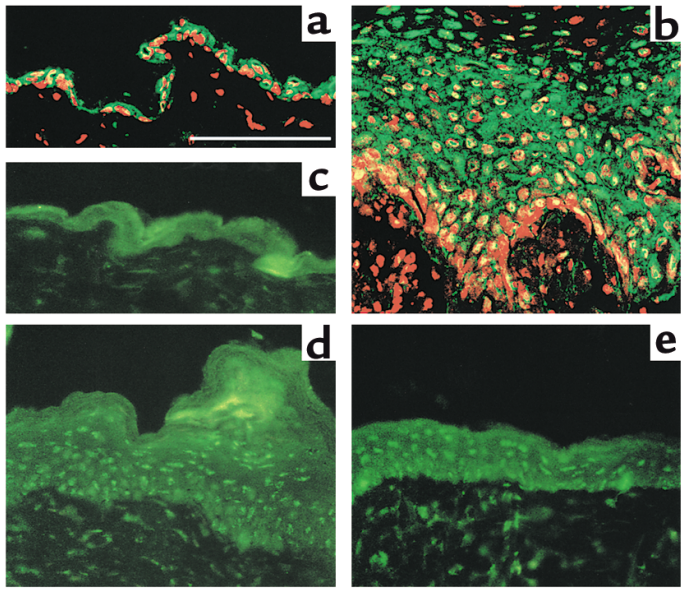

Figure 2

Distribution and activation of $42^{\text {mapk }}$ in phenotypically normal and hyperproliferative transgenic mouse epidermis. ( $\mathbf{a}$ and $\mathbf{b}$ ) Antibody to total MAPK. (c-e) Antibody to activated MAPK. Hyperproliferative $\beta 1$ transgenic mouse epidermis 7 days after wounding ( $b$ and $\mathbf{e}$ ) or unwounded transgenic mouse skin (a). Phenotypically normal epidermis (c) and psoriatic lesion (d) of $\alpha 6 \beta 1$ transgenic mouse. Red staining in $\mathbf{a}$ and $\mathbf{b}$ is nuclear dye, Topro III. Scale bar: $50 \mu \mathrm{m}$.

mAb13 and also upon attachment to laminin 5, which is mediated by the $\alpha 6 \beta 4$ and $\alpha 3 \beta 1$ integrins. MAPK activation was observed in cells plated on laminin 5 under conditions in which binding via the $\alpha 3 \beta 1$ laminin receptor was inhibited with the $\beta 1$-specific antibody P5D2, thereby confirming that attachment to laminin via $\alpha 6 \beta 4$ was sufficient to trigger MAPK activation (Figure 3a). MAPK activation by cell attachment was transient, phosphorylation of the enzyme being highest after 30 minutes and then gradually decreasing to reach background levels after 4 hours (ref. 4; data not shown). These results indicate that activation of MAPK in human keratinocytes is integrin dependent, even in the presence of a high concentration of EGF.

To test whether integrins expressed by suprabasal keratinocytes were able to signal to MAPK, we used a line of keratinocytes isolated from the skin of an inv $\beta 1$ transgenic mouse (8). When held in suspension, these cells, like normal human keratinocytes, are induced to undergo terminal differentiation: expression of the endogenous integrins is downregulated while expression of involucrin and the $\beta 1$ transgene is induced (8). The transgenic integrin is human and can therefore be distinguished from the endogenous mouse integrins using species-specific antibodies (8). The cells were held in suspension for 16 hours and then plated onto fibronectin or the human $\beta 1$-specific antibodies $\mathrm{mAb} 13$ and P5D2 (Figure 3b). As observed in primary human keratinocytes (compare Figure 3a with 3b) MAPK activity was low in transgenic keratinocytes in suspension but increased when the transgenic integrin was ligated by ECM or anti- $\beta 1$ antibody (Figure $3 \mathrm{~b}$ ). Thus $\beta 1$ integrins expressed on the surface of terminally differentiating keratinocytes can signal through MAPK.
Increased production of IL-1 $\alpha$ by keratinocytes expressing suprabasal integrins. Although MAPK activation in vivo correlated with suprabasal integrin expression in hyperproliferative mouse and human epidermis (Figures 1 and 2) and suprabasal integrins were able to activate MAPK (Figure 3b), constitutive activation of MAPK was not observed in phenotypically normal transgenic mice expressing suprabasal integrins (Figure 2, a and c). We therefore sought to identify an additional signal that might be required for MAPK activation. Given that elevated expression of growth factors and proinflammatory cytokines is a feature of psoriasis (20), we examined whether suprabasal integrin expression correlated with altered cytokine production. We examined four proinflammatory cytokines known to be synthesized by keratinocytes (21): IL-1 $\alpha$, IL-1 $\beta$, TNF- $\alpha$, and IL-6.

We compared keratinocytes isolated from a wild-type mouse with keratinocytes isolated from a transgenic mouse expressing the $\alpha 2$ and $\beta 1$ integrin subunits under the control of the involucrin promoter (Figure 4). We measured cytokine production by preconfluent keratinocytes cocultured overnight with a $3 \mathrm{~T} 3$ feeder layer, keratinocytes held in suspension for 8 or 24 hours to stimulate terminal differentiation, and keratinocytes induced to differentiate in suspension for 8 hours and then replated on type IV collagen to ligate endogenous and transgenic $\alpha 2 \beta 1$. In all experimental situations, keratinocytes expressing suprabasal $\alpha 2 \beta 1$ secreted three- to fourfold the IL- $1 \alpha$ into the medium (Figure 4a) and had larger intracellular stores of IL-1 $\alpha$ (Figure $4 \mathrm{~b}$ ) than did wild-type keratinocytes. Suspension-induced differentiation increased the amount of

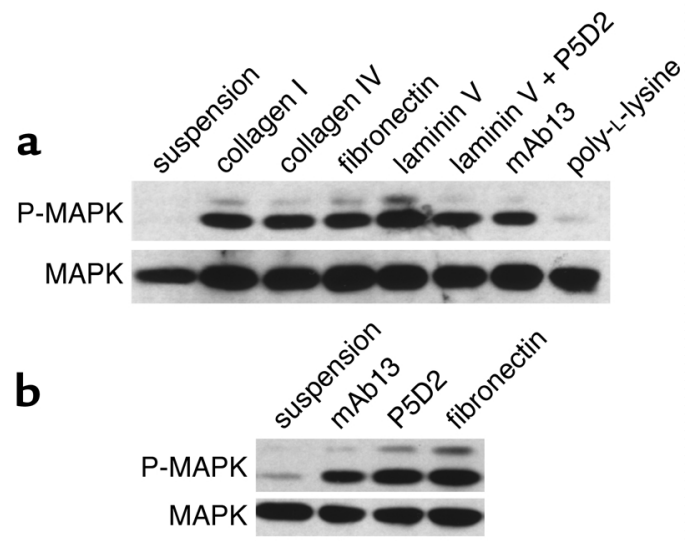

Figure 3

Integrin-mediated MAPK phosphorylation. Western blot analysis of MAPK phosphorylation: upper panels show threonine/tyrosine phosphorylation of ERK1/2 visualized with a phosphorylation-specific antibody; lower panels show the same blots incubated with an antibody against total ERK2 as a loading control. (a) Human keratinocytes in FAD medium containing $10 \mathrm{ng} / \mathrm{ml}$ EGF were held in suspension or plated for 30 minutes onto dishes coated with poly-L-lysine or the ECM proteins and antibodies shown. (b) Inv $\beta 1$ mouse keratinocytes were cultured in suspension for 12 hours to induce terminal differentiation and transgene expression and then held in suspension or plated for 30 minutes in the presence of $10 \mathrm{ng} / \mathrm{ml}$ EGF onto fibronectin or the human $\beta 1$-specific antibodies mAb13 and P5D2. 
a

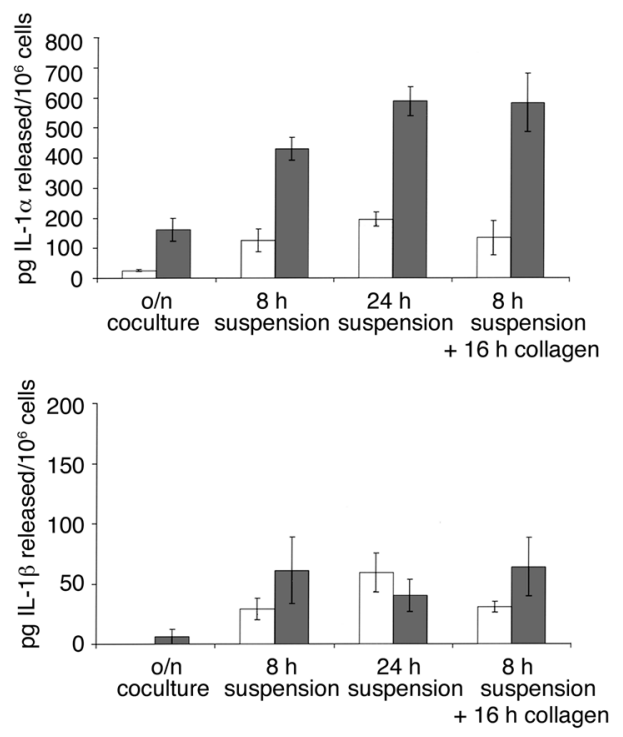

b
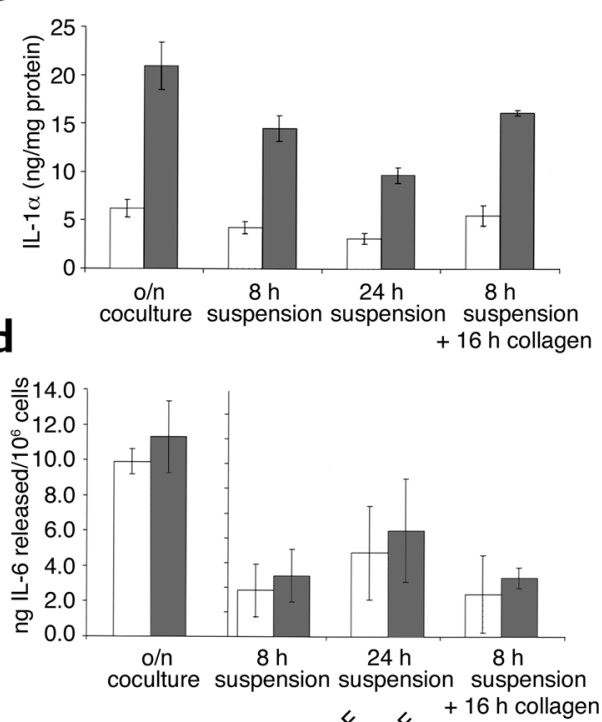

e

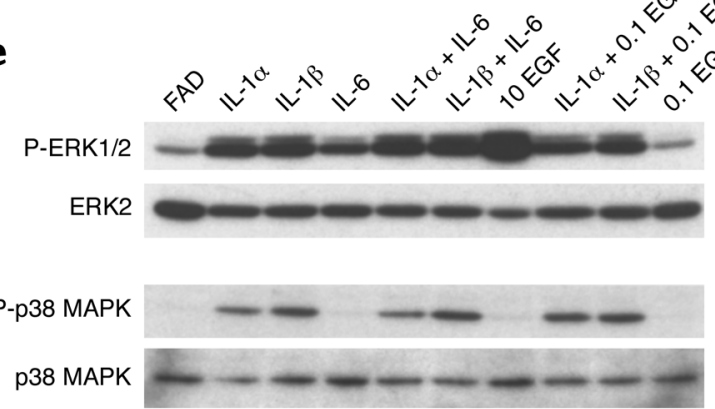

\section{Figure 4}

Proinflammatory cytokine production and cytokine-induced MAPK phosphorylation. Secretion of IL-1 $\alpha$ (a), IL-1 $\beta$ (c), and IL-6 (d) by nontransgenic (open bars) and $\alpha 2 \beta 1$ transgene-expressing (filled bars) mouse keratinocytes. Intracellular IL-1 $\alpha$ levels are shown in $\mathbf{b}$. In the case of overnight keratinocyte/feeder cocultures (o/n coculture), cytokine secreted represents the total produced by both cell types, whereas intracellular levels were measured in keratinocytes alone. (e) Western blot analysis of ERK1/2 and p38 MAPK phosphorylation (P-ERK1/2 and P-p38 MAPK, respectively) and total ERK2 and p38 MAPK. Preconfluent human keratinocytes had feeders removed and were starved overnight before a 15 -minute stimulation with medium alone (FAD) or medium supplemented with IL- $1 \alpha / \beta$, IL-6, or EGF alone or in combinations. IL- $1 \alpha / \beta$ and IL- 6 were added to a final concentration of $10 \mathrm{ng} / \mathrm{ml}$ while EGF was added to $10 \mathrm{ng} / \mathrm{ml}$ when the only cytokine present (10 EGF) or $0.1 \mathrm{ng} / \mathrm{ml}$ when used in combination with other cytokines (0.1 EGF).

IL-1 $\alpha$ released by transgenic keratinocytes, and this increase was independent of ligation of $\alpha 2 \beta 1$ (Figure 4a; compare 24-hour suspension with 8-hour suspension +16 -hour attachment to collagen). Intracellular stores of IL-1 $\alpha$ decreased with time in suspension and were restored by replating on collagen (Figure 4b).

The difference in cytokine production between transgenic and control keratinocytes was specific for IL- $1 \alpha$. IL-1 $\beta$ release was induced equally in suspended transgenic and control keratinocytes (Figure 4c), and the greater amount released by transgenic compared with control keratinocytes after replating on collagen was not statistically significant (Figure 4c). The amount of intracellular IL-1 $\beta$ was below the detection limit of the assay. There was no difference in IL- 6 release between transgenic and control cultures (Figure 4d), and intracellular IL-6 levels mirrored the levels secreted (data not shown). The amount of IL-6 released in overnight cocultures was an order of magnitude greater than the amount released by keratinocytes in suspension or suspended and replated, and this probably reflects IL-6 release by the feeder cells (22). TNF- $\alpha$ production (secreted and intracellular) in transgenic and wild-type keratinocytes was below $25 \mathrm{ng} / \mathrm{ml}$, the limit of sensitivity of the assay (data not shown).

We next tested whether IL-1 $\alpha$ activated MAPK in adherent human keratinocytes, using $10 \mathrm{ng} / \mathrm{ml}$ EGF as a positive control (Figure 4e). IL- $1 \alpha$ and $\beta$ activated ERK, although to a lesser extent than $10 \mathrm{ng} / \mathrm{ml}$ EGF did. IL-6 also had a stimulatory effect, but it was lower than the effect of IL-1. No further activation was observed with higher concentrations of the cytokines. There was no synergy in ERK activation between IL-1 and IL-6, nor between IL- 1 and EGF ( 0.1 or $1 \mathrm{ng} / \mathrm{ml}$ EGF; Figure $4 \mathrm{e}$ and data not shown). As reported previously (23), IL-1 $\alpha$ and $\beta$, but not IL-6 or EGF, activated p38MAPK (Figure 4e).

We conclude that suprabasal integrin expression by keratinocytes is correlated with increased IL- $1 \alpha$ release and that IL-1 can activate ERK in keratinocytes. 

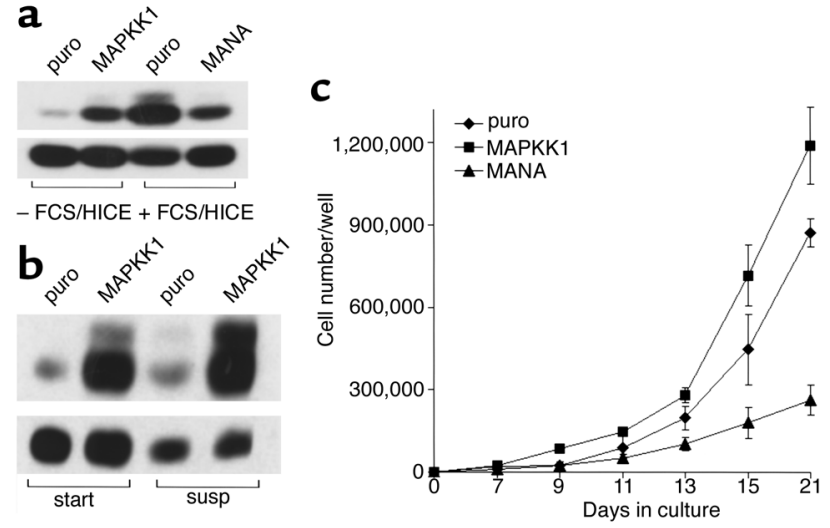

Figure 5

Effect of MAPK activity on keratinocyte proliferation. (a and $\mathbf{b}$ ) Western blot detection of MAPK phosphorylation (upper panels) and total MAPK (lower panels) as described in the legend to Figure 3. (a) Adherent keratinocytes infected with empty vector puro, MAPKK1, or MANA were starved overnight and lysed 10 minutes after addition of fresh medium with or without FCS/HICE. (b) Keratinocytes infected with puro or MAPKK1 were trypsinized and held in suspension for 15 minutes (start) or 24 hours (susp). (c) Growth curves: 500 (puro, MAPKK1) or 1,000 (MANA) keratinocytes were plated per 35-mm dish (to achieve equal numbers of adherent cells; ref. 4) and cultured for 21 days. Triplicate dishes were harvested on the days shown. Error bars = SD.

Effect of MAPK activation on keratinocyte proliferation. To modulate MAPK activity independently of integrins and growth factors, we introduced mutants of MAPK kinase 1 into primary human keratinocytes by means of retroviral infection, as described previously (4). Expression of a MAPK kinase 1 construct containing two activating point mutations (MAPKK1; ref. 11) led to constitutive activation of the MAPK cascade, as indicated by an increased level of phosphorylated MAPK in both adherent (Figure 5a) and suspended (Figure 5b) keratinocytes. The elevated level of MAPK phosphorylation achieved by expression of MAPKK1 in the absence of growth factors was lower than that in control (transduced with empty vector, puro) adherent cells stimulated for 10 minutes with medium containing FCS/HICE (Figure 5a), but was stable for at least 24 hours in adherent and suspended keratinocytes (Figure $5 \mathrm{~b}$ and data not shown). Thus, introduction of MAPKK1 led to a sustained, submaximal activation of MAPK in adherent and suspended keratinocytes. Expression of a dominant negative mutant of MAPK kinase 1 , MANA, caused reduced MAPK activation after stimulation of starved cells with medium containing FCS/HICE (Figure 5a), similar to the effect of MANA on attachmentinduced MAPK phosphorylation (4).

We next used cells infected with constitutively active (MAPKK1) or dominant negative (MANA) mutants of MAPK kinase 1 to investigate the role of this signaling path-

Figure 6 way in proliferation of primary human keratinocytes. Expression of MAPKK1 led to an increased growth rate compared with the empty vector control, puro, whereas introduction of MANA caused a reduction of cell proliferation (Figure 5c).

Although constitutive MAPK activity is sufficient to transform mammalian cell lines $(11,24)$, primary human keratinocytes expressing MAPPK1 still underwent growth arrest in suspension. The proportion of Sphase cells fell from $21.5 \pm 3.5 \%$ in adherent cultures of cells expressing puro to $1.8 \pm 0.9 \%$ after 24 hours in suspension. In cells expressing MAPKK1, it fell from $27.7 \pm 1.2 \%$ to $1.0 \pm 0.14 \%$. Data are mean values from triplicate samples $\pm \mathrm{SD}$.

Effect of MAPK activation on suspension-induced terminal differentiation. In keratinocytes, loss of integrin engagement is a strong trigger for the onset of terminal differentiation (3) and simultaneously results in disabled signal transduction via MAPK (Figure 3a). To examine the effects of MAPK activation on terminal differentiation, human keratinocytes infected with puro or MAPKK1 were placed in suspension for different lengths of time (Figure 6). In cells infected with puro, expression of involucrin, cornifin, and transglutaminase 1 was induced, with the proportion of cells expressing each protein increasing from 8 to 24 hours in suspension; this is revealed as an increase in the size of the cell peak with high fluorescence and a corresponding decrease in the cell peak with low fluorescence. Keratinocytes infected with MAPKK1 also showed an increase in expression of the differentiation markers; however, the number of cells expressing each protein (cell peak with high fluorescence) was always much lower than in puroinfected cells (Figure 6). Thus forced activation of MAPK signaling delayed the onset of suspensioninduced keratinocyte differentiation.

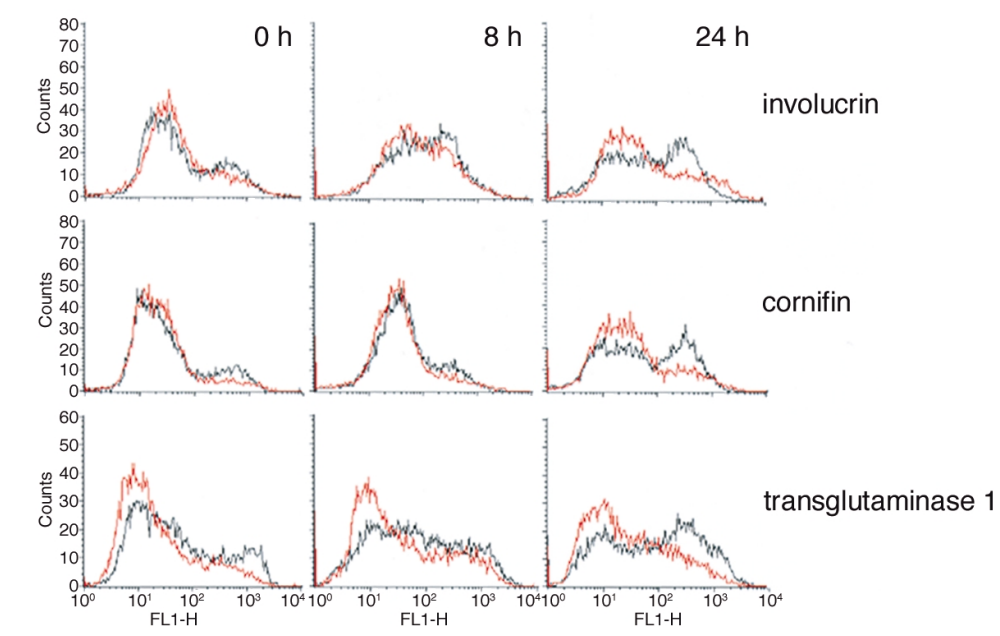

Effect of constitutive MAPK activation on suspension-induced terminal differentiation. Flow cytometry profiles of keratinocytes fixed immediately after trypsinization ( 0 hours) or after 8 or 24 hours in suspension. Cells were labeled with antibodies to the differentiation markers indicated. Black lines: Cells expressing empty vector puro; red lines: cells expressing MAPKK1. 


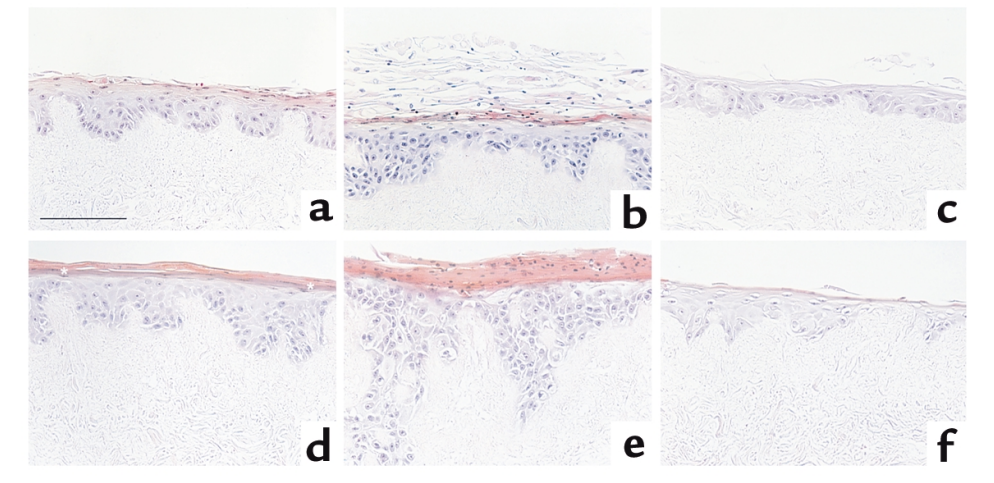

Figure 7

Effects of constitutive activation or inhibition of MAPK on reconstituted epidermis. Human keratinocytes infected with puro ( $\mathbf{a}$ and $\mathbf{d})$, MAPKK1 (b and $\mathbf{e}$ ), or MANA ( $\mathbf{c}$ and $\mathbf{f}$ ) were cultured for $11(\mathbf{a}-\mathbf{c})$ or $14(\mathbf{d}-\mathbf{f})$ days on dead, de-epidermized dermis. Asterisks in $\mathbf{d}$ indicate cells in granular layer. Scale bar: $100 \mu \mathrm{m}$.

Effect of MAPK activation on proliferation and differentiation of reconstituted epidermis. When keratinocytes are seeded on dead, de-epidermized dermis and cultured at the air-liquid interface, they are able to reconstitute an epidermis with many of the characteristics of the normal tissue (ref. 13 and references cited therein). We compared epidermis reconstituted by keratinocytes infected with puro, MAPKK1, and MANA. At day 11 of culture, keratinocytes infected with puro had formed a stratified epithelium with a single basal cell layer and several suprabasal layers, the uppermost of which had started to form the dead, cornified layers characteristic of the final stage of keratinocyte terminal differentiation (Figure 7a). By day 14, the epidermis formed by puro-infected cells had slightly increased in thickness, and a largely anucleate cornified layer had developed with an underlying granular layer, thus bearing a close resemblance to normal epidermis (Figure 7d).

Keratinocytes expressing MAPKK1 formed an epidermis that was already slightly thicker than the puro controls at day 11 and by day 14 showed pronounced thickening and elongated dermal projections (Figure 7, b and e). Immunostaining with an antibody to type IV collagen demonstrated that the basement membrane was intact (data not shown) and so the downgrowths of keratinocytes were not invasive fronts, but probably reflected the topology of the dermal substrate. In sharp contrast to cells expressing puro, there was no granular layer and nuclei were retained in the thick cornified layer that had formed by day 14. Keratinocytes expressing MANA gave rise to a thinner epidermis than the puro controls in which the basal cell layer was not clearly distinguishable and which by day 14 had only a thin, anucleate cornified layer (Figure 7, c and f).

To analyze proliferation in day 11 cultures, we used immunostaining with an antibody against the Ki 67 proliferation-associated antigen. The average number of Ki 67-positive cells per high-power field in 11-day cultures of keratinocytes expressing puro was $16.4 \pm$ 1.6 ; in keratinocytes expressing MAPKK1, it was $29.6 \pm$
4.6; and in keratinocytes expressing MANA, it was $9.9 \pm 2.4$. The average number of basal cells per field was 115 for puro, 134 for MAPKK1-, and 99 for MANAexpressing keratinocytes. Even allowing for differences in the cellularity of the basal layer, introduction of MAPKK1 led to an almost twofold increase in the number of Ki 67-positive cells, whereas MANA caused a reduction in proliferation. In the puroand MANA-expressing cultures, the majority of Ki 67-positive cells were in the basal layer, whereas in the MAPKK1-expressing cultures, suprabasal Ki 67-positive cells were also present (data not shown).

MAPPK1 expression delayed the onset of involucrin expression in reconstituted epidermis (Figure 8). In cultures of keratinocytes expressing puro, basal cells were involucrin negative (red) and all suprabasal layers were involucrin positive (green or yellow in Figure 8a). In contrast, cultures infected with MAPKK1 contained several suprabasal involucrin-negative cell layers (Figure $8 b$, red) and involucrin expression was confined to a small compartment of cells directly beneath the cornified layer (Figure 8b, green or yellow). In epidermis reconstituted by MANA-expressing cells, the expression pattern of involucrin was similar to the puro control, in that all the suprabasal layers were positive; however, there were more involucrin-positive basal cells in the MANA cultures (Figure 8c).

\section{Discussion}

Using the nuclear localization and phosphorylation of MAPK as a readout for its activity $(17,25)$, we found that in hyperproliferative wounded or psoriatic epidermis, but not in normal epidermis, MAPK was activated in basal and suprabasal keratinocytes. The suprabasal cells with nuclear MAPK staining were cells that expressed $\beta 1$ integrins. Ligation of suprabasal integrins activated MAPK, thus establishing that the receptors were capable of signal transduction. Although there is no evidence for suprabasal accumulation of the major basement membrane proteins in hyperproliferative epidermis (6), certain suprabasal integrins could potentially be ligated in vivo by minor collagens, such as type XIII collagen (26).

A second mechanism by which suprabasal integrins could activate MAPK is through stimulating keratinocytes to release IL-1 $\alpha$. The $\alpha 2 \beta 1$ transgenic keratinocytes in culture synthesized and released more IL- $1 \alpha$ than control keratinocytes did, independent of integrin ligation, and IL- $1 \alpha$ activated keratinocyte MAPK. In fibroblasts, IL- $1 \alpha$ expression is increased by changes in cell shape, which can be induced by exposure to soluble or insoluble $\alpha 5 \beta 1$ integrin ligand (27), and in monocytes, ligation of $\alpha \mathrm{M} \beta 2$ or $\alpha X \beta 2$ stimulates production of IL-1 $\beta$ (28). The concept that changes in cell shape could affect IL-1 release is consistent with the increase in IL-1 $\alpha$ release when transgenic keratinocytes were placed in suspension (Figure 4a). In 


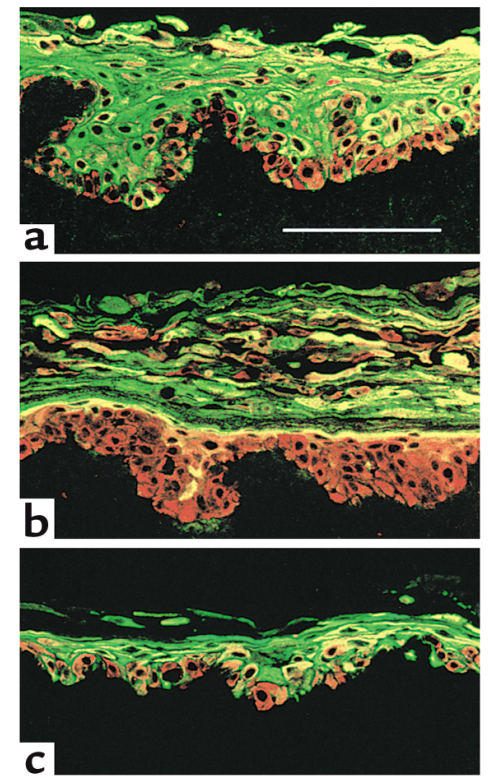

Figure 8

Effects of constitutive activation or inhibition of MAPK on involucrin expression in reconstituted epidermis. Keratinocytes infected with empty vector puro (a), MAPKK1 (b), or MANA (c) were cultured on dead, de-epidermized dermis for 11 days and stained with antibodies against keratin 14 (expressed by all cells; red) and involucrin (green). Scale bar: $100 \mu \mathrm{m}$.

addition, mechanical deformation of human keratinocytes leads to rapid release of IL- $1 \alpha$, and this has been proposed to explain why psoriatic lesions are most commonly in locations where the skin is subject to repetitive stretch or trauma (29).

Even though suprabasal integrins were capable of activating MAPK directly or via stimulating release of IL-1 $\alpha$, examination of phenotypically normal transgenic epidermis established that suprabasal integrins do not activate MAPK constitutively. One explanation is that keratinocyte responsiveness to IL- $1 \alpha$ does not correlate directly with the amount of IL- $1 \alpha$ released. Keratinocytes express an IL-1 receptor antagonist and the nonsignaling type 2 IL-1 receptor, both of which decrease responsiveness to IL-1 (30). In addition, there are double paracrine pathways of growth regulation involving keratinocytes and dermal fibroblasts, such that keratinocyte release of IL-1 induces fibroblasts to produce KGF and IL-6, and fibroblasts reduce the level of keratinocyte IL- 1 in the medium by a process of internalization $(22,31)$. IL-1 $\alpha$ production by keratinocytes induces a dermal mononuclear infiltrate, leading to release of further cytokines and growth factors (32), and this could account for the inflammation seen in the lesions of transgenic mice expressing suprabasal integrins (7).

Although we ruled out any difference in expression of IL-1 $\beta$, IL- 6 , and TNF- $\alpha$ between wild-type and transgenic keratinocytes in culture, it would obviously be premature to conclude that IL- $1 \alpha$ is the only cytokine to be selectively upregulated when integrins are expressed suprabasally. The recent finding that the phorbol ester TPA, which can activate MAPK via protein kinase C (33), selectively induces suprabasal proliferation in transgenic epidermis (34) opens the way for an in vivo survey of all growth factors and cytokines that might contribute to the activation of MAPK in this model of psoriasis, one of the candidate cytokines being IL-12 (35). Equally, MAPK is certainly not the only signaling pathway that is altered in psoriasis; for example, IL-1 activates the NF- $\mathrm{KB}$ pathway, which is of pivotal importance in cutaneous inflammation $(36,37)$ and in normal epidermal homeostasis (38). Finally, the changes in epidermal gene expression observed in response to MAPK activation imply altered activity of a host of transcriptional regulators (38-40), and these remain to be examined in integrin transgenic mice.

MAPK activation contributed to epidermal hyperproliferation. Expression of MAPKK1 resulted in an increased growth rate of keratinocytes both under conventional culture conditions (Figure 5) and in reconstituted epidermis (Figure 7); conversely, inhibition of MAPK signaling by expression of MANA reduced cell proliferation. Previous work has shown that although MANA increases the proportion of transit-amplifying cells, MAPKK1 has no effect on the proportion of stem cells in culture (4). The stimulation of growth by MAPKK1 may therefore occur by delaying the onset of terminal differentiation, observed both in suspension and in reconstituted epidermis (Figures 6 and 8). An increase in the proliferative potential of the transitamplifying compartment would have a dramatic effect on the number of keratinocytes: one additional round of cell division would double the number of cells in the epidermis. Interestingly, expansion of the transitamplifying compartment is thought to occur in psoriasis, leading to elongation of the rete ridges (41), and, in epidermis reconstituted by MAPKK1 expressing cells, we observed more pronounced keratinocyte downgrowths than in controls (Figure 7e).

Whereas MAPK activity seems to stimulate proliferation in many cell types $(9,24)$ and is also implicated in senescence and apoptosis (42), its role in the regulation of differentiation is more variable. In MDCK cells, features of differentiation are suppressed (43), whereas in PC12 cells, MAPK stimulates neurite outgrowth (11). In skeletal muscle cells, MAPK activity inhibits early stages of differentiation but stimulates later phases (44). Activated MAPK delayed, but did not inhibit, the onset of keratinocyte differentiation; suspended keratinocytes transduced with MAPKK1 did not divide (data not shown), suggesting that they were committed to terminal differentiation (3). The histological changes in reconstituted epidermis after constitutive activation of MAPK mimicked the appearance of psoriatic lesions (45): the epidermis was thickened, with more proliferating cells, a reduction in the granular layer and a highly thickened, nucleated cornified layer. Whereas these changes reflect the consequences of pathological MAPK activation in vivo, a certain physiological level of MAPK activity is required in order to form a normal stratified epithelium, because epidermis reconstituted by MANA-expressing cells was hypocellular, with decreased proliferation and incomplete terminal differentiation. 
In mammalian cell lines, constitutive activation of the MAPK pathway is sufficient for cell transformation (11, 24). Although activation of MAPK in keratinocytes stimulated proliferation and delayed differentiation cells expressing MAPKK 1 did not exhibit two key properties of transformed cells: they did not proliferate in suspension, and they showed no invasiveness in organotypic cultures. This is consistent with psoriatic lesions of the skin being fully reversible and psoriasis itself not increasing the risk of skin tumor development (45). It is likely that the pathological activation of the MAPK pathway that has been observed in various tumors (46) is due to mutational changes in upstream regulating molecules. Both in wound healing and in psoriasis, there seems to be a transient and reversible upregulation of MAPK activity that is mediated through upstream regulation by integrins and growth factors. This makes pharmacological interference with MAPK signaling a promising approach for the treatment of psoriasis.

\section{Acknowledgments}

We are very grateful to everyone who provided advice or reagents, particularly Marina Haitas-Haase and Karin Hartmann. This work was supported by the Imperial Cancer Research Fund and by a grant to I. Haase from the Deutsche Forschungsgemeinschaft (Ha 2623/1-1).

1. Watt, F.M. 1998. Epidermal stem cells: markers, patterning and the control of stem cell fate. Philos. Trans. R. Soc. Lond. B. Biol. Sci. 353:1-7.

2. Fuchs, E. 1990. Epidermal differentiation. Curr. Opin. Cell Biol. 2:1028-1035.

3. Jones, P.H., and Watt, F.M. 1993. Separation of human epidermal stem cells from transit amplifying cells on the basis of differences in integrin function and expression. Cell. 73:713-724.

4. Zhu, A.J., Haase, I., and Watt, F.M. 1999. Signaling via $\beta 1$ integrins and mitogen-activated protein kinase determines human epidermal stem cell fate in vitro. Proc. Natl. Acad. Sci. USA. 96:6728-6733.

5. Levy, L., Broad, S., Diekmann, D., Evans, R.D., and Watt, F.M. 2000. $\beta 1$ integrins regulate keratinocyte adhesion and differentiation by distinct mechanisms. Mol. Biol. Cell. 11:453-466.

6. Watt, F.M., and Hertle, M.D. 1994. Keratinocyte integrins. In The keratinocyte handbook. I.M. Leigh, E.B. Lane, and F.M. Watt, editors. Cambridge University Press. Cambridge, United Kingdom. 153-164.

7. Carroll, J.M., Romero, M.R., and Watt, F.M. 1995. Suprabasal integrin expression in the epidermis of transgenic mice results in developmental defects and a phenotype resembling psoriasis. Cell. 83:957-968.

8. Romero, M.R., Carroll, J.M., and Watt, F.M. 1999. Analysis of cultured keratinocytes from a transgenic mouse model of psoriasis: effects of suprabasal integrin expression on keratinocyte adhesion, proliferation and terminal differentiation. Exp. Dermatol. 8:53-67.

9. Seger, R., and Krebs, E.G. 1995. The MAPK signaling cascade. FASEB J. 9:726-735

10. Alessi, D.R., et al. 1994. Identification of the sites in MAP kinase kinase-1 phosphorylated by $\mathrm{p}^{74^{\mathrm{raf}-1}}$. EMBOJ.13:1610-1619.

11. Cowley, S., Paterson, H., Kemp, P., and Marshall, C.J. 1994. Activation of MAP kinase kinase is necessary and sufficient for PC12 differentiation and for transformation of NIH 3T3 cells. Cell. 77:841-852.

12. Gandarillas, A., and Watt, F.M. 1997. c-Myc promotes differentiation of human epidermal stem cells. Genes Dev. 11:2869-2882.

13. Rikimaru, K., Molès, J.-P., and Watt, F.M. 1997. Correlation between hyperproliferation and suprabasal integrin expression in human epidermis reconstituted in culture. Exp. Dermatol. 6:214-221.

14. Hogquist, K.A., et al. 1991. Generation of monoclonal antibodies to murine IL-1 $\beta$ and demonstration of IL-1 in vivo. J. Immunol. 146:1534-1540.

15. Thacher, S.M., and Rice, R.H. 1985. Keratinocyte-specific transglutaminase of cultured human epidermal cells: relation to cross-linked envelope formation and terminal differentiation. Cell. 40:685-695.

16. Fujimoto, W., Nakanishi, G., Arata, J., and Jetten, A.M. 1997. Differential expression of human cornifin $\alpha$ and $\beta$ in squamous differentiating epithelial tissues and several skin lesions. J. Invest. Dermatol. 108:200-204.

17. Lenormand, P., Brondello, J.M., Brunet, A., and Pouyssegur, J. 1998. Growth factor-induced $\mathrm{p} 42 / \mathrm{p} 44$ MAPK nuclear translocation and retention requires both MAPK activation and neosynthesis of nuclear anchoring proteins. $J$. Cell Biol. 142:625-633.

18. Renshaw, M.W., Ren, X.-D., and Schwartz, M.A. 1997. Growth factor activation of MAP kinase requires cell adhesion. Cell. 16:5592-5599.

19. Lin, T.H., Chen, Q., Howe, A., and Juliano, R.L. 1997. Cell anchorage permits efficient signal transduction between ras and its downstream kinases. J. Biol. Chem. 272:8849-8852.

20. Krueger, J.G., and Gottlieb, A.B. 1994. Growth factors, cytokines and eicosanoids. In Psoriasis. L. Dubertret, editor. ISED Publishing Co. Brescan, Italy. 18-28.

21. Kondo, S. 1999. The roles of keratinocyte-derived cytokines in the epidermis and their possible responses to UVA-irradiation. J. Investig. Dermatol. Symp. Proc. 4:177-183.

22. Maas-Szabowski, N., Stark, H.-J., and Fusenig, N.E. 2000. Keratinocyte growth regulation in defined organotypic cultures through IL-1-induced keratinocyte growth factor expression in resting fibroblasts. J. Invest. Dermatol. 114:1075-1084.

23. Ichijo, H. 1999. From receptors to stress-activated MAP kinases. Oncogene. 18:6087-6093.

24. Mansour, S.J., et al. 1994. Transformation of mammalian cells by constitutively active MAP kinase kinase. Science. 265:966-970.

25. Brunet, A., et al. 1999. Nuclear translocation of p42/p44 mitogen-activated protein kinase is required for growth factor-induced gene expression and cell cycle entry. EMBOJ. 18:664-674.

26. Peltonen, S., et al. 1999. A novel component of epidermal cell-matrix and cell-cell contacts: transmembrane protein type XIII collagen. J. Invest. Dermatol. 113:635-642.

27. Kheradmand, F., Werner, E., Tremble, P., Symons, M., and Werb, Z. 1998. Role of Rac1 and oxygen radicals in collagenase- 1 expression induced by cell shape change. Science. 280:898-902.

28. Rezzonico, R., Chicheportiche, R., Imbert, V., and Dayer,J.-M. 2000. Engagement of CD11b and CD11c $\beta 2$ integrin by antibodies or soluble CD23 induces IL- $1 \beta$ production on primary human monocytes through mitogenactivated protein kinase-dependent pathways. Blood. 95:3868-3877.

29. Lee, R.T., et al. 1997. Mechanical deformation promotes secretion of IL- $1 \alpha$ and IL-1 receptor antagonist. J. Immunol. 159:5084-5088.

30. Kupper, T.S., and Groves, R.W. 1995. The interleukin-1 axis and cutaneous inflammation. J. Invest. Dermatol. 105:62S-66S.

31. Boxman, I.L.A., Ruwhof, C., Boerman, O.C., Löwick, C.W.G.M., and Ponec, M. 1996. Role of fibroblasts in the regulation of proinflammatory interleukin IL-1, IL-6 and IL-8 levels induced by keratinocyte-derived IL-1. Arch. Dermatol. Res. 288:391-398.

32. Groves, R.W., Mizutani, H., Kieffer, J.D., and Kupper, T.S. 1995. Inflammatory skin disease in transgenic mice that express high levels of interleukin $1 \alpha$ in basal epidermis. Proc. Natl. Acad. Sci. USA. 92:11874-11878.

33. Schonwasser, D.C., Marais, R.M., Marshall, C.J., and Parker, P.J. 1998. Activation of the mitogen-activated protein kinase/extracellular signal-regulated kinase pathway by conventional, novel, and atypical protein kinase $\mathrm{C}$ isotypes. Mol. Cell. Biol. 18:790-798.

34. Owens, D.M., and Watt, F.M. 2001. Influence of $\beta 1$ integrins on epidermal squamous cell carcinoma formation in a transgenic mouse model: $\alpha 3 \beta 1$, but not $\alpha 2 \beta 1$, suppresses malignant conversion. Cancer Res. 61:5248-5254.

35. Hong, K., Chu, A., Lúdvíksson, B.J., Berg, E.L., and Ehrhardt, R.O. 1999. Il12 , independently of IFN- $\gamma$, plays a crucial role in the pathogenesis of a murine psoriasis-like skin disorder. J. Immunol. 162:7480-7491.

36. Robert, C., and Kupper, T.S. 1999. Inflammatory skin diseases, T cells, and immune surveillance. N. Engl.J. Med. 341:1817-1828.

37. Murphy, J.-E., Robert, C., and Kupper, T.S. 2000. Interleukin-1 and cutaneous inflammation: a crucial link between innate and acquired immunity. J. Invest. Dermatol. 114:602-608.

38. Kaufman, C.K., and Fuchs, E. 2000. It's got you covered: NF-KB in the epidermis. J. Cell Biol. 149:999-1004.

39. Eckert, R.L., Crish, J.F., Banks, E.B., and Welter, J.F. 1997. The epidermis: genes on - genes off. J. Invest. Dermatol. 109:501-509.

40. Sinha, S., Degenstein, L., Copenhaver, C., and Fuchs, E. 2000. Defining the regulatory factors required for epidermal gene expression. Mol. Cell. Biol. 20:2543-2555.

41. Iizuka, H., Ishida-Yamamoto, A., and Honda, H. 1996. Epidermal remodelling in psoriasis. Br. J. Dermatol. 135:433-438.

42. Cobb, M.H. 1999. MAP kinase pathways. Prog. Biophys. Molec. Biol. 71:479-500.

43. Schramek, H., Feifel, E., Healy, E., and Pollack, V. 1997. Constitutively active mutant of the mitogen-activated protein kinase kinase MEK1 induces epithelial dedifferentiation and growth inhibition in madin-darby canine kidney-C7 cells. J. Biol. Chem. 272:11426-11433.

44. Bennett, A.M., and Tonks, N.K. 1997. Regulation of distinct stages of skeletal muscle differentiation by mitogen-activated protein kinases. Science. 278:1288-1291.

45. Lever, W.F., and Schaumburg-Lever, G. 1983. Histopathology of the skin. J.B. Lippincott Co. Philadelphia, Pennsylvania, USA. 136-163.

46. Sebolt-Leopold, J.S., et al. 1999. Blockade of the MAP kinase pathway suppresses growth of colon tumors in vivo. Nat. Med. 5:810-816. 